\title{
Complex Addition Labelling With Gaussian Integers
}

\author{
G.Jayalalitha ${ }^{1}$, S.Kavitha ${ }^{2}$ \\ ${ }^{1}$ Professor, Department of Mathematics, Vels University, Chennai, Tamilnadu, India. \\ Email: ragaji94@yahoo.com \\ ${ }^{2}$ Research scholar, Bharathiar University, Coimbatore, Tamilnadu, India. \\ Email: kavithasundaram55@gmail.com
}

\begin{abstract}
Aim of this paper is to introduce the Gaussian integers Z[i] to the graph labelling. Define a complex number set as $\gamma_{p-1}, p \geq 2$ and Complex Addition Labelling. Some families of graphs which may be satisfied the Complex Addition Labelling and bounds of vertices and edges of the Complex Addition Labelling are discussed.
\end{abstract}

Keywords: Gaussian Integers, Ordering Set, Complex Addition Labelling, Complete Graph, Cycle Graph with Chord

\section{INTRODUCTION}

All graphs in this paper are finite, simple, undirected and without loops unless otherwise stated. This paper addresses labelling graphs in such a way that the vertex labels are the real and imaginary part of the incident edge labels for every vertex. In order to view the impression of a graph labelling(Joseph A. Gallian 2014) to Gaussian integers, initially let us define what is mean by "the first n Gaussian integers".

In section 2, explicate a $\gamma_{p-1}, p \geq 2$ ordering set of the Gaussian integers that allows to linearly order the Gaussian integers, definitions and Examples of Complex Addition Labelling graphs. In section 3 , investigate the families of the Graphs which satisfy the Complex Addition Labelling.

\section{BACKGROUND AND PRELIMINARIES}

2.1. Definition of Basics and Gaussian Integers

This paper starts with some admissible backdrop on Gaussian integers to deliver a foot base of our work (Kavitha $\mathrm{S}$ and Jayalalitha G,2017 and Rosen K.H,2011 ). The structure of the complex numbers is $a+b i$, where $a, b \in$ $Z$ and $i^{2}=-1$ is called the Gaussian integers, denoted by $Z[i]$. The unit in the Gaussian integers is one of $\pm 1, \pm i$. An associate of Gaussian integers $\alpha$ is $u$. $\alpha$ where $\mathrm{u}$ is a Gaussian unit. The norm of a Gaussian integer $a+b i$, denoted by $N(a+b i)$, is given by $a^{2}+b^{2}$. A Gaussian integer is even if it is divisible by $1+i$ and odd otherwise. This is because Gaussian integers with even norms are divisibly by $1+i$. The view is to expand the investigation of graph labelling to Gaussian integers. Because the Gaussian integers are not totally ordered, it is a must to give an appropriate definition of Gaussian integers first which we take (Hunter Lehmann, Andrew Park 2011, Klee. S, et al.,2016). Here propose the following ordering of the Gaussian integers denoted by $\gamma_{p-1}, p \geq 2$ is defined as

Table 1. Construction of Gaussian Integers

\begin{tabular}{|c|c|c|c|c|c|c|c|c|}
\hline$\gamma_{p-1}$ & 2 & 3 & 4 & $\cdot$ & $\cdot$ & $\cdot$ & $p-1$ & $P$ \\
\hline 1 & $1+2 i$ & $1+3 i$ & $1+4 i$ & $\cdot$ & $\cdot$ & $\cdot$ & $\cdot$ & $1+p i$ \\
\hline 2 & - & $2+3 i$ & $2+4 i$ & $\cdot$ & $\cdot$ & $\cdot$ & $\cdot$ & $2+p i$ \\
\hline 3 & - & - & $3+4 i$ & $\cdot$ & $\cdot$ & $\cdot$ & $\cdot$ & $3+p i$ \\
\hline$\cdot$ & $\cdot$ & $\cdot$ & $\cdot$ & $\cdot$ & $\cdot$ & $\cdot$ & $\cdot$ & $\cdot$ \\
\hline$\cdot$ & $\cdot$ & $\cdot$ & $\cdot$ & $\cdot$ & $\cdot$ & $\cdot$ & $\cdot$ & $\cdot$ \\
\hline$\cdot$ & $\cdot$ & $\cdot$ & $\cdot$ & $\cdot$ & $\cdot$ & $\cdot$ & $\cdot$ & $(p-2)+p i$ \\
\hline$p-2$ & $\cdot$ & $\cdot$ & $\cdot$ & $\cdot$ & $\cdot$ & $\cdot$ & $(p-2)+(p-1) i$ & $(p-1)+p i$ \\
\hline$p-1$ & - & - & - & - & - & - & - & $(p-1)$ \\
\hline
\end{tabular}

In general the ordering set $\gamma_{p-1}$ is in the upper triangular matrix form of order $(p-1) X(p-1)$ and the Gaussian Integers take $\operatorname{Re}(Z)<\operatorname{Im}(Z)$. The Gaussian integers take as a set of integers $\{1+2 \mathrm{i}\},\{1+2 \mathrm{i}, 1+3 \mathrm{i}, 2+3 \mathrm{i}\},\{1+2 \mathrm{i}, 1+3 \mathrm{i}, 1+4 \mathrm{i}, 2+3 \mathrm{i}, 2+4 \mathrm{i}, 3+4 \mathrm{i}\}$, 
That it takes the number of Gaussian integers in each set as $\left\{1,3,6,10,15,21,28, \ldots \ldots, \frac{p(p-1)}{2}\right\}$.

\section{Definition 2.2}

Let $G(V, E)$ be a $(p, q)$ graph and $p \geq 2$. A function $\mathrm{f}$ is called Complex Addition Labelling of a graph $\mathrm{G}$, if $f: V(G) \rightarrow\{1,2,3, \ldots p\}$ is injective and the induced function $f^{*}: E(G) \rightarrow\left[\gamma_{p-1}\right]$ is defined as

$$
f^{*}(e=u v)=\left\{\begin{array}{l}
f(u)+\text { if }(v) \text { if } u<v \\
f(v)+i f(u) \text { if } v<u
\end{array}\right. \text { is bijective. }
$$

A graph which admits Complex Addition Labelling is called a Complex Addition Graph.

\section{Definition 2.3}

A graph is Complete if every two distinct vertices in the graph are adjacent.

\section{Definition 2.4}

Let $G=\left(V(G), E(G), I_{G}\right)$ and $\mathrm{H}=\left(V(H), E(H), I_{H}\right)$ be two graphs. A graph isomorphism from $\mathrm{G}$ to $\mathrm{H}$ is a $\operatorname{pair}(\varnothing, \theta)$, where $\emptyset: V(G) \rightarrow V(H)$ and $\theta: E(G) \rightarrow E(H)$ are bijections with the property that $I_{G}(e)=$ $\{u, v\}$ if and only if $I_{H}(\theta(e))=\{\varnothing(u), \emptyset(v)\}$. If $(\varnothing, \theta)$ is a graph isomorphism, the pair of inverse mappings $\left(\varnothing^{-1}, \theta^{-1}\right)$ is also a graph isomorphism.

\section{Theorem 2.5}

If two graphs $\mathrm{G}$ and $\mathrm{H}$ are isomorphic, then they have the same order and the same size, and the degrees of the vertices of $\mathrm{G}$ are the same as the degrees of the vertices of $\mathrm{H}$.

\section{Definition 2.6} set C.

A Chord of a graph Cycle $\mathrm{C}$ is an edge not in the edge set of $\mathrm{C}$ whose end points lie in the vertex

\subsection{Examples of some Complex Addition Labelling:}

Taking the first three ordering sets $\gamma_{1}, \gamma_{2}$ and $\gamma_{3}$ of Gaussian Integers [Table 2,3,4] some examples of graphs which admits the Complex Addition Labelling by using the Gaussian integers of the above three sets are shown here.[Figure.1,2,3]

Table.2. $\gamma_{1}$ ordering set $(p=2)$

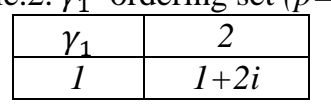

Table.3. $\gamma_{2}$ ordering set $(p=3)$

\begin{tabular}{|c|c|l|}
\hline$\gamma_{2}$ & 2 & 3 \\
\hline 1 & $1+2 i$ & $1+3 i$ \\
\hline 2 & - & $2+3 i$ \\
\hline
\end{tabular}

Table.4. $\gamma_{3}$ ordering set $(p=4)$

\begin{tabular}{|c|c|c|c|}
\hline$\gamma_{3}$ & 2 & 3 & 4 \\
\hline 1 & $1+2 i$ & $1+3 i$ & $1+4 i$ \\
\hline 2 & - & $2+3 i$ & $2+4 i$ \\
\hline 3 & - & - & $3+4 i$ \\
\hline
\end{tabular}

1

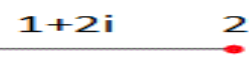




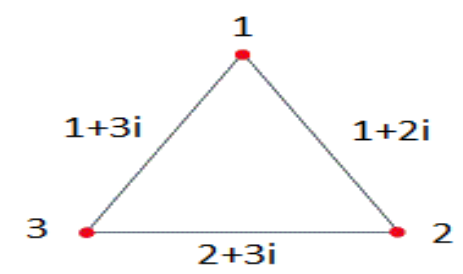

Figure.2:Complex Addition

$\operatorname{Graph}(\mathrm{p}=2)$

Figure.1: Complex Addition

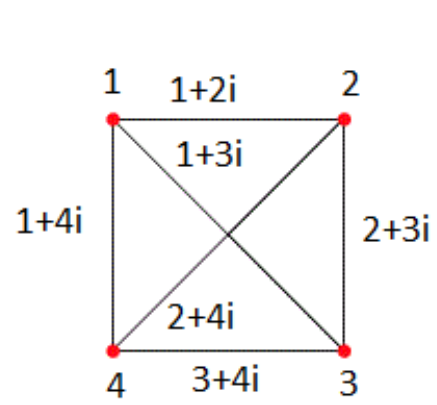

$\operatorname{Graph}(\mathrm{p}=3)$

Figure.3: Complex Addition $\operatorname{Graph}(\mathrm{p}=4)$

\section{THEOREMS ON COMPLEX ADDITION LABELLING}

Based on Table.1 and definition of 2.2 construction of a Complex Addition Labelling for several classes of graphs are discussed.

\section{Theorem 3.1}

Complex Addition Labelling graph $G(p, q)$ has $\frac{p(p-1)}{2}$ edges.

\section{Proof}

Let $G(p, q)$ is a Complex Addition Labelling. By definition 2.2, $f^{*}$ is bijective, number of edges in $\mathrm{G}$ is equal to the number of Gaussian integers in $\gamma_{p-1}$ ordering set and it is in a triangular form [Table 1].

First row of the $\gamma_{p-1}$ ordering set in Table. 1 has $p-1$ Gaussian integers, second row has $p-2$ Gaussian integers and the last row has one Gaussian integer. Now the number of total Gaussian integers is equal to sum of first $p-1$ natural integers. This implies that $G(p, q)$ has $\frac{p(p-1)}{2}$ edges. Hence the proof.

\section{Theorem 3.2}

The Graph $G(p, q)$ is not a Complex Addition Labelling Graph with at least one vertex of degree $(p-2)$, for all $p \geq 2$

\section{Proof}

Let $G(p, q)$ is a Complex Addition Labelling. By definition 2.2 of the Complex Addition Labelling, each vertex has degree $p-1$.If at least one vertex has degree $p-2$ then it contradicts to the definition.

Hence it is not a Complex Addition Labelling.

\section{Theorem 3.3}

The Complete graph $K_{n}$ admits a Complex Addition Labelling for all $n \geq 2$. 
International Journal of Research in Advent Technology, Vol.7, No.1, January 2019 E-ISSN: 2321-9637

\section{Available online at www.ijrat.org}

\section{Proof}

In every complete graph $K_{n}$, the number of edges is $\frac{n(n-1)}{2}$ and each vertex is adjacent to all other vertices.[Figure.4]

Let $V_{1}=1$.Then by the definition 2.3 of a Complete Graph, $V_{1}$ is adjacent to all other vertices and the edges are labelled with the first row Gaussian integers of the $\gamma_{p-1}$ ordering set.

Let $V_{1}=2$ and its edges are labelled with the second row Gaussian integers of $\gamma_{p-1}$ ordering set.

Continue this process till to the last vertex it has the edge labelling by all other rows of $\gamma_{p-1}$ ordering set. So every complete graph $K_{n}$ admits Complex Addition Labelling. Hence the proof.

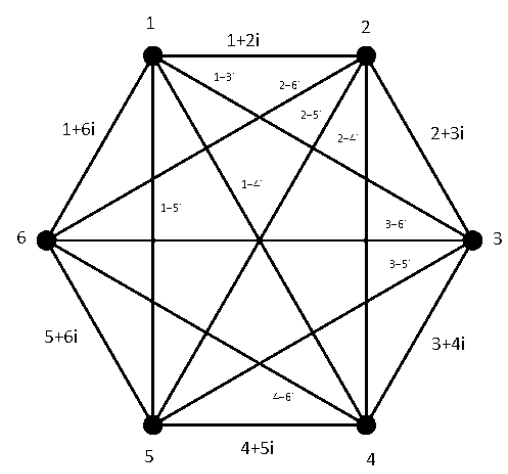

Theorem 3.4

Fig.4.The Complete Graph $K_{6}$

Let $G=(p, q)$ be a Complex Addition graph, then

$\sum_{i} \operatorname{Re}\left(e_{i}\right)=\frac{1}{6} p\left(p^{2}-1\right) \quad$ and $\quad \sum_{i} \operatorname{Im}\left(e_{i}\right)=\frac{1}{3} p\left(p^{2}-1\right)$

Proof

Let $G=(p, q)$ be a Complex Addition graph. The number of the edges in the graph $\mathrm{G}$ is the same as the number of edges in the $\gamma_{p-1}$ set. By adding the real and imaginary parts of the Gaussian integers in $\gamma_{p-1}$ ordering set, we get

Sum of the real parts $=1+(1+2)+(1+2+3)+(1+2+3+4)+\ldots+(1+2+3+\cdots(p-1))$

$$
\begin{aligned}
& =1+\frac{2 \cdot(2+1)}{2}+\frac{3 \cdot(3+1)}{2}+\frac{4 \cdot(4+1)}{2}+\cdots+\frac{(p-1) \cdot((p-1)+1)}{2} \\
& =\frac{1}{2}(1 \cdot 2+2 \cdot 3+3 \cdot 4+4 \cdot 5+\cdots+(p-1) \cdot p) \\
& =\frac{1}{2}\left(\frac{p\left(p^{2}-1\right)}{3}\right) \\
& =\frac{1}{6} p\left(p^{2}-1\right)
\end{aligned}
$$

Hence $\sum_{i} \operatorname{Re}\left(e_{i}\right)=\frac{1}{6} p\left(p^{2}-1\right)$

Sum of the imaginary parts $=1.2+2.3+3.4+\cdots+(p-1) \cdot p$ 


$$
=\frac{1}{3} p\left(p^{2}-1\right)
$$

Hence $\sum_{i} \operatorname{Im}\left(e_{i}\right)=\frac{1}{3} p\left(p^{2}-1\right)$

\section{Theorem 3.5}

Complex Addition Labelling graph $G=(p, q)$ admits isomorphism for $p \geq 5$

\section{Proof}

If two graphs $\mathrm{G}$ and $\mathrm{H}$ are isomorphic, then they have the same order and the same size, and the degrees of the vertices of $\mathrm{G}$ are the same as the degrees of the vertices of $H$ (Theorem 2.5).By this necessary condition for two graphs to be isomorphic Complex Addition Labelling admits isomorphism.

The graphs $\mathrm{G}$ and $\mathrm{H}$ in Figure.5 are isomorphic; in fact, the function $\emptyset: V(G) \rightarrow V(H)$ defined by $\emptyset(1)=u_{1}, \varnothing(2)=u_{2}, \emptyset(3)=u_{3}, \emptyset(4)=u_{4}$ and $\emptyset(5)=u_{5}$ is an isomorphism (Figure .5)

$\mathrm{G}$ :

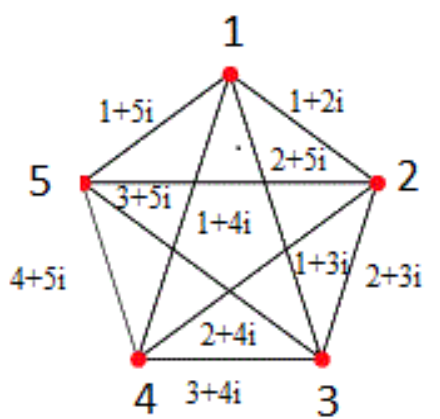

$\mathrm{H}$ :

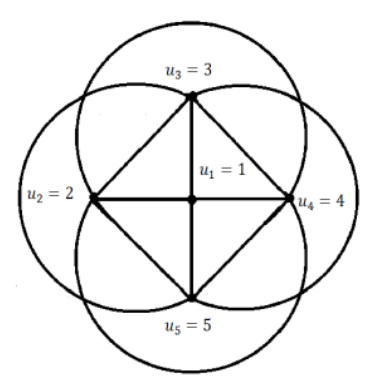

Fig.5. Isomorphic graphs $\mathrm{G}$ and $\mathrm{H}$

\section{REFERENCES}

[1] Hunter Lehmann, Andrew Park, Prime labelling of small trees with the Gaussian integers, Rose-Hulman Undergrad. Math J. (2015) staff.seattleu.edu/klees/web/SmallTrees.pdf,inpress.

[2] Joseph A. Gallian, A dynamic survey of graph labelling, Electron. J. Combin.21 (2014) 43. Dynamic Survey 6 (electronic)

[3] Kavitha S and Jayalalitha G, Prime Labelling with Spiral Gaussian integers International Journal of Pure and Applied Mathematics(IJPAM),Volume 117 No.20 2017,209-217.

[4] Klee. S, et al., Prime labelling of families of trees with Gaussian integers, AKCE International Journal of Graphs and Combinatorics (2016), http://dx.doi.org/10.1016/j.akcej.2016.04.001

[5] Rosen K.H, Elementary Number theory and Its Applications, Addison-wesley,2011. 